\title{
Triggering on electrons, photons, tau leptons, jets and energy sums at HL-LHC with the upgraded CMS Level-1 Trigger
}

\author{
Pallabi Das* \\ on behalf of the CMS Collaboration \\ Department of Physics, Princeton University, \\ Princeton, New Jersey 08544, USA \\ E-mail: pdas@princeton.edu, pallabi.das@cern.ch
}

From the year 2026 onwards, the LHC experiments will operate in the high-luminosity mode (HL-LHC) with 5-7.5 times the design instantaneous luminosity. The Phase-2 upgrade of the Compact Muon Solenoid (CMS) detector aims to mitigate degradation in detector performance and high pile-up rates, about 140-200 per event, due to increase in luminosity. The upgrade of the Level-1 (L1) trigger system will include tracking information and subdetector upgrades leading to higher granularity and precision timing information. An overview of the electrons, photons, jets, hadronic taus and energy sums trigger algorithms for the upgraded L1 system is presented, along with their expected performance studied using simulated collision data of the HL-LHC.

*** The European Physical Society Conference on High Energy Physics (EPS-HEP2021), ***

*** 26-30 July $2021 * * *$

*** Online conference, jointly organized by Universität Hamburg and the research center DESY ***

\footnotetext{
${ }^{*}$ Speaker
} 


\section{Introduction}

During HL-LHC [1] operation, the CMS experiment [2] will accumulate over $3 \mathrm{ab}^{-1}$ of collision data, making it possible to carry out high precision measurements of the standard model (SM), as well as searches for new physics beyond SM. To support this ambitious physics program, the trigger and data acquisition systems will be entirely replaced to prepare for the harsh experimental conditions. For the hardware-based L1 trigger that provides the first decision to select interesting events, the upgrade will combine state-of-the-art technology with sophisticated algorithms [3]. Improved physics performance is expected through the addition of tracking and therefore running the Particle Flow (PF) algorithm [4] at L1. In addition, finer granularity and precision timing information from the calorimeters will ensure better object reconstruction, improving the event selection efficiency.

\section{Electrons and photons}

To maintain the calorimetric performance at the HL-LHC, the high-granularity calorimeter or HGCAL will be installed in the endcaps, while the barrel region will continue having both the electromagnetic and hadronic calorimeters (ECAL and HCAL).

In the barrel region $(|\eta|<1.47)$, the granularity will increase 25 times compared to the current configuration, providing improved position resolution. The identification requirements of the electrons/photons are implemented using shower shape variables around a seed crystal in $\eta-\phi$. A ratio of energies within $2 \times 5 / 3 \times 5$ crystal windows provides the best performance. For determining the isolation, $27 \times 27$ crystals around the seed are chosen, corresponding to a $\Delta R$ of $\sim 0.3-0.4$ in offline.

In the endcap $(1.47<|\eta|<3)$ HGCAL uses 3-dimensional information of the shower development to identify clusters originating from electrons or photons. A boosted decision tree (BDT) algorithm is used to distinguish between the electromagnetic deposits from signal and pileup.

Figure 1 illustrates the expected single electron identification efficiencies at HL-LHC.

\section{Jets, hadronic taus and energy sums}

The identification of jets and hadronic taus and the measurement of energy sum quantities will be based on (i) calorimeter information only, (ii) calorimeter objects matched to L1-tracks, (iii) complete PF or PileUp Per Particle Identification (PUPPI) [5] inputs.

The calorimeter-based algorithm uses energy deposits from the barrel, endcap, and forward regions to construct a $7 \times 7$ square geometry of towers to identify a jet. The jet is corrected for the energy reconstruction and pileup, which helps to maintain the low rates even at the HL-LHC conditions. The track-based jets are used to compute scalar energy sum $\left(H_{T}\right)$, vector energy sum $\left(H_{T}^{\text {miss }}\right.$ ), and missing energy (MET or $E_{T}^{\text {miss }}$ ) in the transverse direction. The left plot of Fig. 2 shows that by applying the track quality criteria in the construction of $H_{T}^{\text {miss }}$, the threshold on this variable can be reduced to $290 \mathrm{GeV}$ from $675 \mathrm{GeV}$ for a constant rate. A similar reduction is obtained for track-based $E_{T}^{\text {miss }}$ thresholds. However, the best trigger turn-on performance is obtained for PUPPI $E_{T}^{\text {miss }}$, as shown in the right plot in Fig. 2. PF-jets are also built using PUPPI candidates binned 

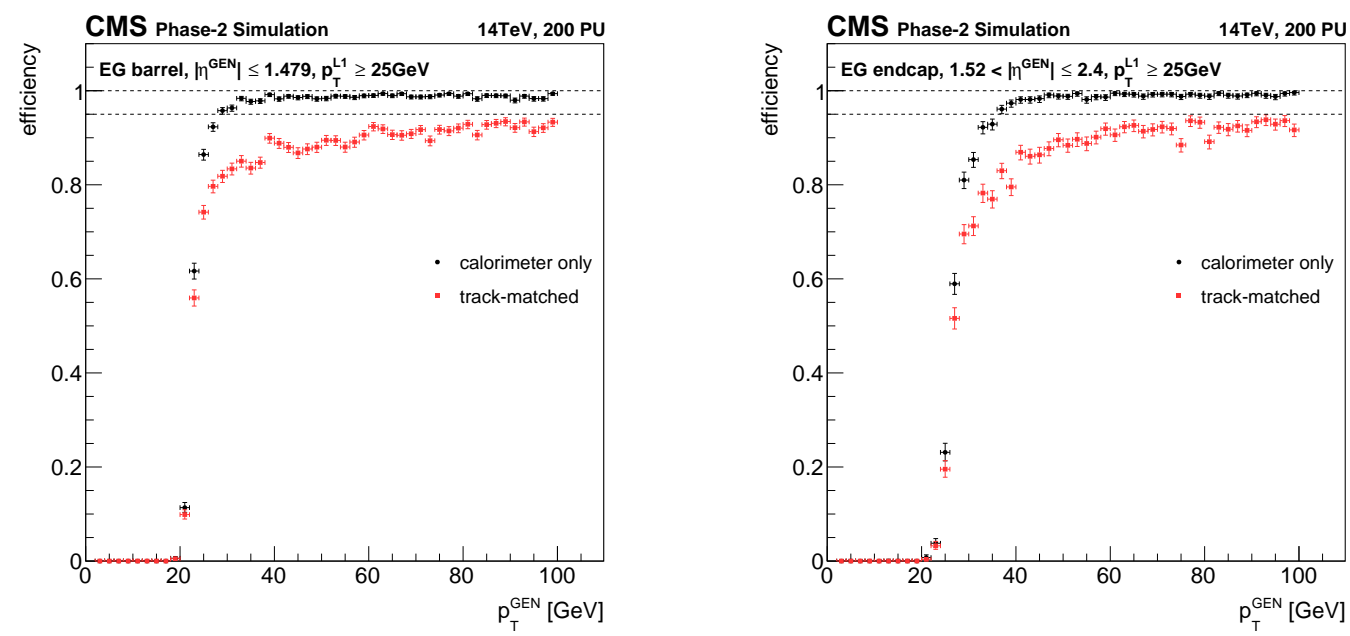

Figure 1: Single electron reconstruction efficiency vs generator-level transverse momentum $\left(p_{T}\right)$ in the barrel (left) and endcap (right) regions. At HL-LHC particle tracks are available up to $|\eta|<2.4$ at L1, which provides a handle to suppress pileup and maintain acceptable trigger rates. The L1 tracks are (i) matched to calorimeter deposits for identifying electrons, (ii) used to define the isolation variable for both electrons/photons. With $\sim 10 \%$ efficiency loss, the single-electron rate reduces by an order of magnitude at low $p_{T}$ with L1-track matching.

into pseudo trigger towers in $\eta-\phi$. It is observed that a $9 \times 9$ geometry of these PF-jets gives the best performance, as larger size contains more energy deposits, and pileup is already suppressed by the PUPPI algorithm. The reconstruction performance thus obtained is similar to anti- $k_{t}$ jets with distance parameter 0.4 (AK4) offline, illustrated in Fig. 3, left plot.
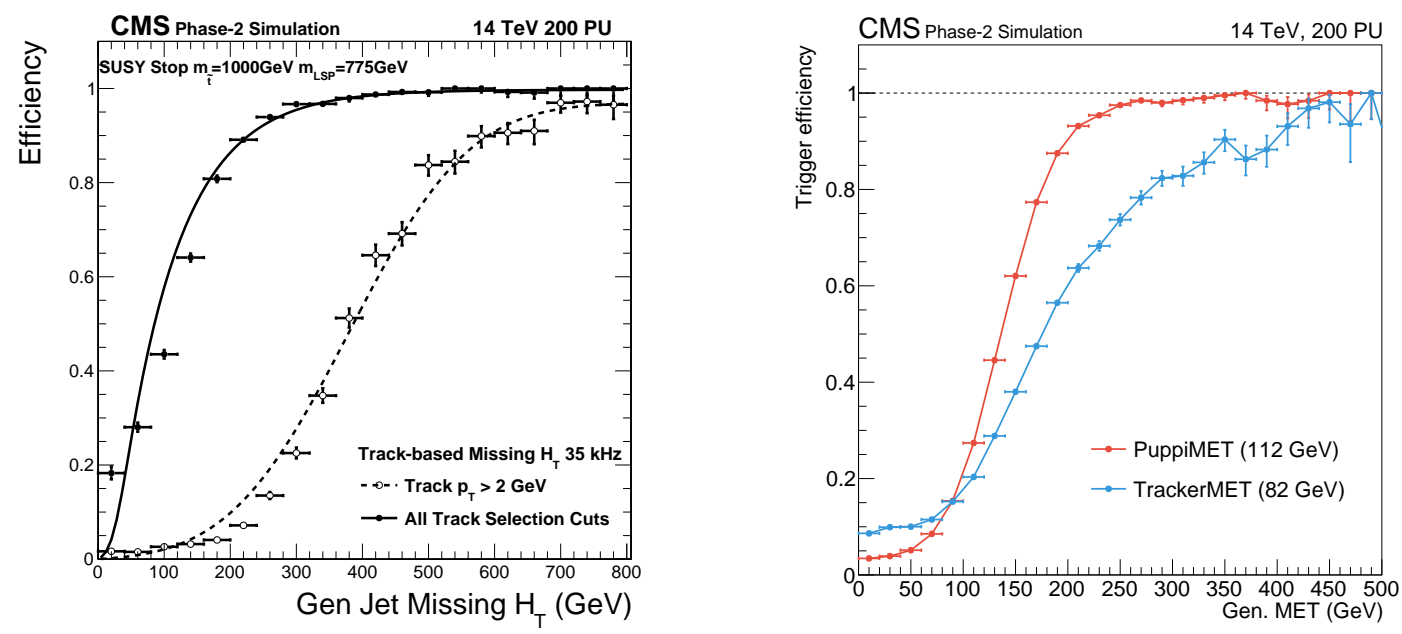

Figure 2: Left: turn-on efficiency for the track-based $H_{T}^{\text {miss }}$ trigger. Right: comparison of track-based and PUPPI $E_{T}^{\text {miss }}$ efficiencies.

The calorimeter-based hadronic tau reconstruction algorithm uses tower information similar to the jet algorithm, along with the energy and pileup corrections. Central $3 \times 5$ towers are used to define the $p_{T}$ while $7 \times 7$ towers are used for isolation. In addition to this, an HGCAL-based algorithm is developed, using a BDT to further optimize the tau identification in the endcap. On 
the other hand, the track $+e / \gamma$ reconstruction first constructs a track-only object and then adds neutral energy by employing calorimeter information. Finally, the PF-based reconstruction takes either PF or PUPPI candidates as input and a neural network $(\mathrm{NN})$ algorithm identifies the tau candidates. This approach mostly relies on event-level quantities, effectively improving overall selection efficiency. The right plot in Fig. 3 shows the direct comparison of the single-tau trigger efficiencies of the three approaches as a function of the generator-level tau $p_{T}$, for a fixed rate.
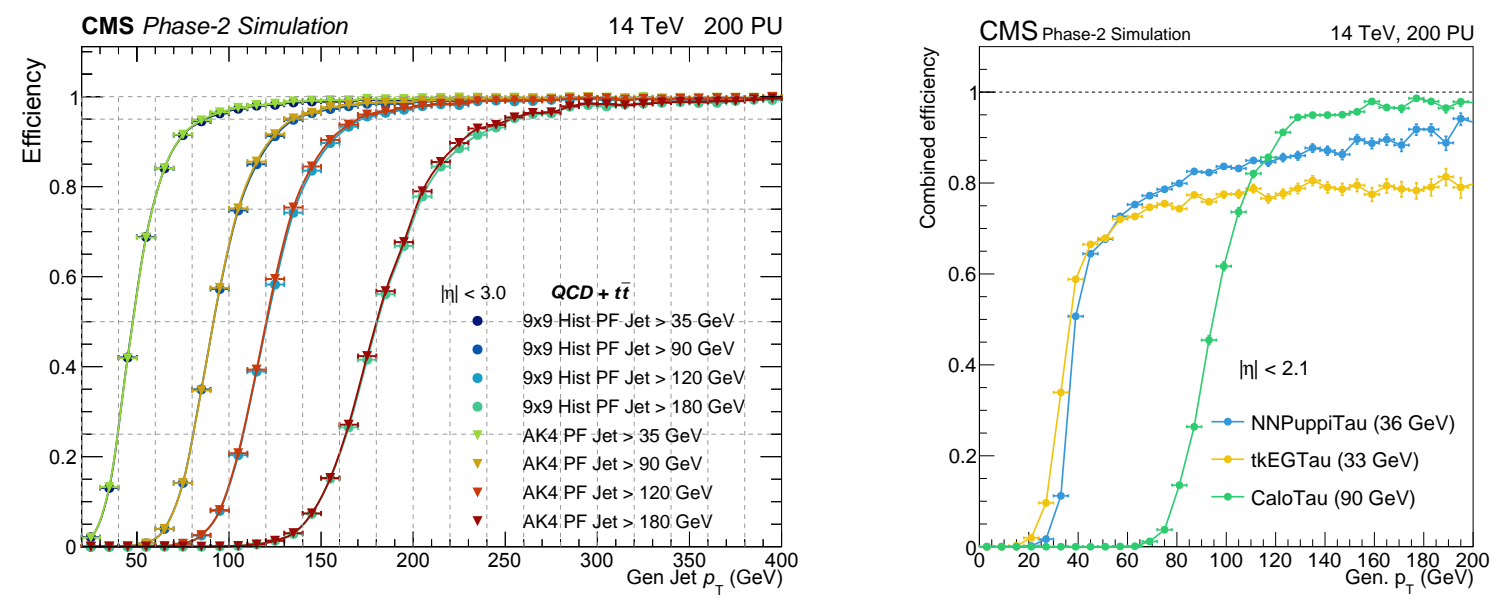

Figure 3: Left: HLT vs offline PF-jets. Right: improved trigger turn-on for track-based and PF-based tau identification compared to simple calo-based algorithm.

\section{Conclusion}

Not only advanced hardware installation but also sophisticated algorithms for object reconstruction at L1 will ensure smooth physics performance during Phase-2 operations. Some of the improved algorithms for electrons, photons, jets, taus, energy sum quantities have been discussed briefly. Many of these algorithms have already been implemented in firmware applications. However, further developments are in progress to better exploit detector upgrades and features.

\section{References}

[1] Aberle, O. et al., "High-Luminosity Large Hadron Collider (HL-LHC): Technical design report", CERN-2020-010.

[2] CMS Collaboration, "The CMS experiment at the CERN LHC", JINST 3 (2008) S08004.

[3] CMS Collaboration, "The Phase-2 Upgrade of the CMS Level-1 Trigger”, CERN-LHCC2020-004.

[4] CMS Collaboration, "Particle-flow reconstruction and global event description with the CMS detector", JINST 12 (2017) P10003.

[5] D. Bertolini, P. Harris, M. Low, and N. Tran, "Pileup Per Particle Identification", JHEP 10 (2014) 059. 